\title{
Increased of serum IL-I $\beta$ and MDA in diabetic and hypertensive retinopathies
}

\begin{abstract}
Hyperglycemia of diabetes and hypertension are associated with vascular complications leading to retina lesion. Both conditions lead to blindness, and the inflammation and oxidative stress have a pivotal role in the pathological consequences. We aimed to compare biomarkers of inflammation in both kinds of retinopathy. Biomarker of oxidative stress, malondialdehyde (MDA), and proinflammatory cytokines, IL-1 $\beta$ and IL- 6 , were evaluated comparatively. MDA was quantified using the TBARS assay, and the levels of cytokines were measured using ELISA. The results showed that diabetic and hypertensive retinopathy increase serum MDA and IL-1 $\beta$ in comparison to healthy control. However, the levels of IL$1 \beta$ and MDA observed in diabetic retinopathy were comparable to that seen in hypertensive pathology in the eye. The concentration of IL-6 was not changed when patients with retinopathy (diabetic or hypertensive) were compared with healthy control. Patients with or without diabetic retinopathy showed similar results about MDA levels. These data suggest that MDA and IL- $1 \beta$ showed a systemic response and both could be used as biomarkers of inflammation in eyes and as a follow-up for therapeutic control.
\end{abstract}

Volume 5 Issue 6 - 2017

\author{
Helder Costa Drumond, Caroline Maria \\ Oliveira Volpe, Rafael Mourão Agostini, José \\ Augusto Nogueira-Machado \\ Núcleo de Pós-Graduação e Pesquisa, Hospital Santa Casa de \\ Belo Horizonte, Brazil
}

\begin{abstract}
Correspondence: José Augusto Nogueira-Machado, Núcleo de Pós-Graduação e Pesquisa, Hospital Santa Casa de Belo Horizonte, Rua Domingos Vieira 590, Santa Efigênia, 30I50-240, Belo Horizonte, MG, Brazil,Tel 55 3I 32388838,

Email aunog.bh@outlook.com.br
\end{abstract}

Received: November 09, 2017 | Published: December 6, 2017

Keywords: retinopathy, diabetes, hypertension, Il-1 $\beta$, IL-6, cytokines, inflammation

Abbreviations: ELISA, enzyme linked immuno sorbent assay; FA, fluorecente angiography; IL, interleukin; MDA, malondialdehyde; ND, non-diabetic; RP, retinopathy; T2DM, type 2diabetes mellitus; TBARS, thiobarbituric acid reactive substances

\section{Introduction}

Retinopathy (RP) is a microvascular complication induced by diabetes and hypertension. Its onset and progression are associated with chronic hyperglycemia and dysregulated arterial hypertension. In microvascular abnormality, it is observed increased oxidative stress and elevated inflammatory cytokines in the eyes. Diabetic retinopathy is triggered by hyperglycemia of diabetes, however, while in nondiabetic individuals, retinopathy can be triggered by chronic arterial hypertension inducing abnormality in arteriolar retina circulation leading to hypertensive retinopathy. Chronic and noncontrolled hypertension leads to a pathological hypertensive retinopathy and microalbuminuria, ${ }^{1}$ both, diabetic and hypertensive retinopathies constitute inflammatory processes and culminating with blindness. Some biomarkers of inflammation are altered in aqueous humor and serum. It is reported that in type 2 diabetes mellitus (T2DM) patients with retinopathy, VEGF and IL-6 were similar in plasma but increased in aqueous humor. ${ }^{2}$ Superoxide is high in the retinas of diabetic rats and retinal cells incubated in high-glucose media. ${ }^{3,4}$ Reports have been suggested that oxidative stress mediate several diabetic complications, including retinopathy. Chan \& Zhu et al, ${ }^{5,6}$ in a review and meta-analysis study observed a significant association between gestational hypertensive disorders and development of retinopathy of prematurity. High serum level of uric acid correlated with the presence of hypertensive retinopathy, ${ }^{7}$ stroke, diastolic dysfunction and renal abnormality. ${ }^{8-11}$ In spite of several studies on diabetic retinopathy, there are few reports in the literature comparing diabetic and hypertensive retinopathy. We aimed to study the level of a biomarker of oxidative stress (malondialdehyde) and to evaluate cytokines from inflammasome (IL-1 $1 \beta$ ) or activation of NF$\kappa \mathrm{B}$ (IL-6) in T2DM with either hypertensive or diabetic.

\section{Materials and methods}

\section{Study population}

The Ethical Committee from Santa Casa Hospital of Belo Horizonte - Brazil approved this study, and the informed consent was obtained from all participants. Patients suffering from T2DM (diagnosed according to the criteria of the American Diabetes Association) were diagnosed by Endocrinology service of Santa Casa Hospital. Participants were recruited at the Ophthalmology Center of the Santa Casa Hospital, Belo Horizonte, Brazil. Volunteers were within the age range of 30-80years and included individuals presenting T2DM as well as healthy individuals with or without retinopathy. Diabetic and hypertensive retinopathy was diagnosed by Dr. Rafael Agostini, using Fluorescence angiography (FA), Canon CF-1 Retinal Camera. Before the study, all volunteers were submitted to full physical examinations, and detailed evaluations of medical histories and laboratory data were carried out. Subjects presenting dementia, inflammation, infection or malignant disease were excluded from the study, as were pregnant women and individuals with alcohol or tobacco dependency. The studied population comprised four groups for studying, 25 healthy controls, non-diabetic without retinopathy (ND); 26 T2DM patients without retinopathy (T2DM); 41 patients with hypertensive retinopathy (ND with RP); 41patients with diabetic retinopathy (T2DM with RP). Diabetic patients were taking medications such as statins, beta-blockers, besides hypoglycaemic. Patients with hypertensive retinopathy were taking anti-hypertension medications.

\section{Blood collection}

Peripheral venous blood samples were collected in patients by venipuncture technique using vacutainer tubes without anticoagulant to obtain blood serum. The tubes were placed in the refrigerator for 8 hours for clot retraction and then centrifuged. Serum samples were separated and stored at $-80^{\circ} \mathrm{C}$ until required for analysis. 


\section{Quantification of malondialdehyde (MDA) and IL-I $\beta$ and IL-6}

Malondialdehyde (MDA) quantification was performed using the kit for determination of thiobarbituric acid reactive substances and the dosage was performed according to the manufacturer's instructions of the "TBARS (Thiobarbituric Acid Reactive Substances) Assay Kit." The quantification of cytokines IL- $1 \beta$ and IL- 6 were performed by the Enzyme Linked Immuno Sorbent Assay for (ELISA) technique according to the manufacturer's instructions using Human IL- $\beta$ ELISA MAX' ${ }^{\mathrm{TM}}$ Deluxe-Biolegend" and "Human IL-6 ELISA MAX"TM kits.

\section{Statistical analyses}

The Kolmogorov-Smirnov test was used to assess the normal distribution of the continuous variables, and values were expressed as mean \pm standard error. Comparisons between groups were performed using unpaired Student $t$ test. All tests were performed with GraphPad Prism 5 (GraphPad Software, Inc) with the level of significance set at $\mathrm{p}<0.05$.

\section{Results}

Our results with MDA (Table 1), expressed as mmol/L, showed that the values for healthy control were lesser than those observed in patients with diabetic or hypertensive retinopathy $(\mathrm{p}<0.05)$ (line 1 in comparison to lines 2 and 3). MDA levels of T2DM without retinopathy compared to healthy control (line 1 vs 3 ) were significantly different $(\mathrm{p}<0.05)$. However, the presence of retinopathy did not increase the level of MDA in T2DM patients (lines 3 vs 4) ( $>>0.05$ ). The Figure shows the results of quantification of pro inflammatory cytokines. Levels of IL-1 $\beta$ in nondiabetic patients with hypertensive retinopathy is higher than that observed in healthy control $(\mathrm{p}<0.05)$ (panel A). Similar results were found with T2DM patients without and with retinopathy (panel B). The results with IL-6 are shown in the panels C and D. The levels of IL- 6 were not significantly changed in both hypertensive and diabetic retinopathy when compared with respective controls without retinopathy $(\mathrm{p}>0.05)$.

\section{Discussion}

Pathological alteration in the retinal vasculature may suggest systemic complications in the vessel in several others organs. ${ }^{12}$ around $32 \%$ of the patients with hypertension and nephropathy have hypertensive retinopathy. ${ }^{13}$ the pathogenesis of retinopathy is multifactorial, and the molecular mechanisms are still not fully understood. Both diabetic and hypertensive retinopathy appears to induce similar consequences on the eyes. However, there are few reports in the literature comparing diabetic and hypertensive retinopathies. The results presented herein reveal that the retinopathy, diabetic and hypertensive, show increased serum levels of MDA and IL-1 $\beta$ in relation to healthy control (Figure 1). No statistical difference was found for the IL-6 levels. Serum from patients with diabetic retinopathy showed similar levels of MDA in comparison to diabetic patients without retinopathy. It suggests that the inflammation and oxidative stress was not potentiated by retina vascular lesion observed in the retinopathy. This parameter appears to be mainly induced by hyperglycemia of diabetes. The inflammatory process present in retinopathy did not increase the level of MDA besides to that observed with hyperglycemia (Table 1). Hypertensive retinopathy, per si, appears to be decisive in increased levels of serum MDA. The levels of serum MDA in healthy controls with hypertensive retinopathy is greater than that observed in diabetic patients with retinopathy. Proinflammatory cytokines, in contrast, showed similar levels when IL-
$1 \beta$ and IL-6 from patients with diabetic and hypertensive retinopathy were compared $(\mathrm{p}>0.05)$. Oxidative stress is believed to have a pivotal role in neuronal and vascular damage in the retina. Studies on retinopathy are mainly performed using a diabetic model. However, our results, suggest that the oxidative stress evaluated by quantification of MDA can be associated with elevation of IL-1 $\beta$, possibly due to the participation of inflammasome. Activation of ROS production is increased in the eyes of patients with diabetes. ${ }^{14}$ Uric acid crystal and ROS are able to activate inflammasome. It is reported that serum uric acid concentration is associated with hypertensive retinopathy in hypertensive Chinese adults. ${ }^{7}$ Thus, ROS and MDA increased and the possible association between increased IL- $\beta 1$ and inflammasome activation in the eyes with systemic repercussion can be envisaged. Our results with serum IL-6 are in agreements to Fulgencio Cunha et al. ${ }^{2}$ No significant difference between serum IL-6 levels in patients with diabetic or hypertensive retinopathy in comparison to healthy control was found (Figure 1). The present results demonstrated that both diabetic and hypertensive retinopathy increased MDA and IL$1 \beta$ in serum. These results suggest that at least two biomarkers for diabetes or hypertension retinopathies showed similar inflammatory profiles indicating a systemic response from the eyes. Thus, can be hypothesized that retinopathy in consequence of the oxidative and inflammatory systemic mediators could induce or aggravates lesion in other organs.
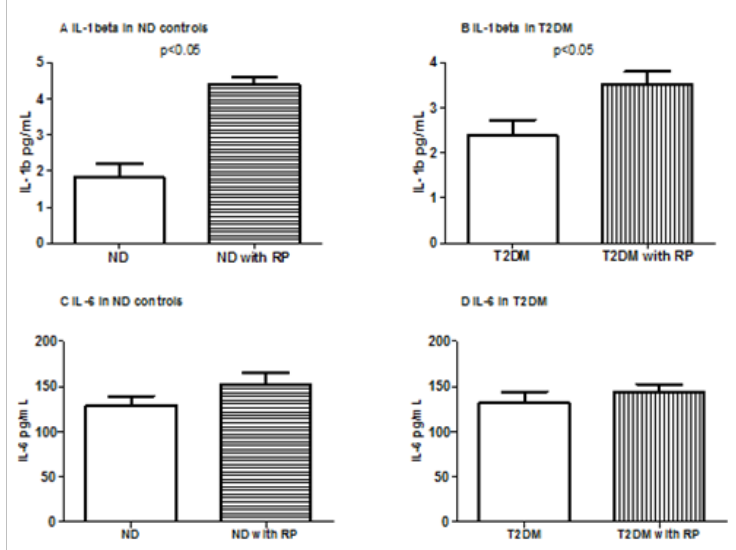

Figure I Serum levels of pro-inflammatory cytokines from non-diabetic (ND) controls and Type 2diabetes mellitus (T2DM) or nondiabetic patients without and with diabetic or hypertensive retinopathy (RP). Panel A and B-Levels of Interleukin Ibeta (IL-Ib); Panel C and D-Levels of IL-6.

Table I Levels of malondialdehyde (MDA) from non-diabetic (ND) controls and Type 2 diabetes mellitus (T2DM) patients or hypertensive patients without and with retinopathy

\begin{tabular}{ll}
\hline Experiments & MDA mmol/L \\
\hline Non-diabetic without retinopathy & $14.7 \pm 2.2$ \\
Non-diabetic with retinopathy & $32.1 \pm 2.9^{*}$ \\
T2DM without retinopathy & $24.1 \pm 2.1^{*}$ \\
T2DM with retinopathy & $24.4 \pm 2.4 \#$ \\
\hline
\end{tabular}

Data shown as mean \pm standard error. ${ }^{*} \mathrm{p}<0.05$ vs Non-diabetic without retinopathy.

\# $\mathrm{p}<0.05$ vs Non-diabetic with retinopathy. 


\section{Acknowledgements}

The authors wish to thank Coordenação de Aperfeiçoamento de Pessoal de Nível Superior (CAPES), Conselho Nacional de Desenvolvimento Científico e Tecnológico (CNPq), Fundação de Amparo à Pesquisa do Estado de Minas Gerais (FAPEMIG) and Rede Mineira de Toxina Terapêutica 26/12 for financial support. The funding bodies played no role in study design, data collection and analysis, or preparation/publication of the manuscript.

\section{Conflicts of interest}

The authors declare that they have no competing interests.

\section{Funding}

None.

\section{References}

1. Karadag B, Ozyigit T, Serindag Z, et al. Blood pressure profile is associated with microalbuminuria and retinopathy in hypertensive nondiabetic patients. Wien Klin Wochenschr. 2017.

2. Fulgencio Cunha AA, Bosco AA, Veloso CA, et al. Suppressive effect of aqueous humor from person with type 2 diabetes with or without retinopathy on reactive oxygen species generation. Diabetes Res Clin Pract. 2013;100(1):69-73.

3. Du Y, Miller CM, Kern TS. Hyperglycemia increases mitochondrial superoxide in retina and retinal cells. Free Radic Biol Med. 2003;35(11):1491-1499.

4. Kowluru RA, Abbas SN. Diabetes-induced mitochondrial dysfunction in the retina. Invest Ophthalmol Vis Sci. 2003;44(12):5327-5334.

5. Chan PY, Tang SM, Au SC, et al. Association of Gestational Hypertensive Disorders with Retinopathy of prematurity: A Systematic Review and Meta-analysis. Sci Rep. 2016;6:30732.
6. Zhu T, Zhang L, Zhao F, et al. Association of maternal hypertensive disorders with retinopathy of prematurity: A systematic review and meta-analysis. PLoS One. 2017;12(4):e0175374.

7. Chen X, Meng Y, Li J, et al. Serum uric acid concentration is associated with hypertensive retinopathy in hypertensive chinese adults. $B M C$ Ophthalmol. 2017;17(1):83.

8. Arriozola-Rodriguez KJ, Serna-Ojeda JC, Martinez-Hernandez VA, et al. Hypertensive Retinopathy as the First Manifestation of Advanced Renal Disease in a Young Patient: Report of a Case. Case Rep Ophthalmol. 2015;6(3):415-419.

9. Kanar BG, Kanar HS, Karatay A, et al. Assessment of left atrium and diastolic dysfunction in patients with hypertensive retinopathy: A real-time three-dimensional echocardiography-based study. Clin Exp Hypertens. 2017;39(8):696-704.

10. Omotoso AB, Kolo PM, Olanrewaju TO, et al. Relationship between retinopathy and renal abnormalities in black hypertensive patients. Clin Hypertens. 2016;22:19.

11. Ong YT, Wong TY, Klein R, et al. Hypertensive retinopathy and risk of stroke. Hypertension. 2013;62(4):706-711.

12. Grunwald JE, Pistilli M, Ying GS, et al. Retinopathy and the risk of cardiovascular disease in patients with chronic kidney disease (from the Chronic Renal Insufficiency Cohort study). Am J Cardiol. 2015;116(10):1527-1533.

13. Malhotra SK, Gupta R, Sood S, et al. Bilateral renal artery stenosis presenting as hypertensive retinopathy and choroidopathy. Indian $J$ Ophthalmol. 2002;50(3):221-223.

14. Sugai M, Ohta A, Ogata Y, et al. Asymmetric dimethylarginine (ADMA) in the aqueous humor of diabetic patients. Endocr J. 2007;54(2):303-309. 